\title{
Assessment of the Hematopoietic Differentiation Potential of Human Pluripotent Stem Cells in 2D and 3D Culture Systems
}

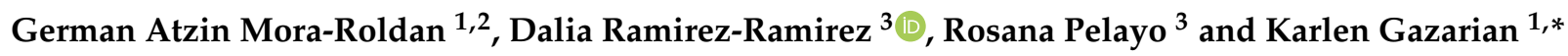 \\ 1 Departamento de Medicina Genómica y Toxicología Ambiental, Instituto de Investigaciones Biomédicas, \\ Universidad Nacional Autónoma de México, Cuidad de México 04510, Mexico; \\ germanmora@iibiomedicas.unam.mx \\ 2 Instituto de Oftalmología Fundación, Conde de Valenciana IAP, Cuidad de Mexico 04510, Mexico \\ 3 Centro de Investigación Biomédica de Oriente, Instituto Mexicano del Seguro Social, Km 4.5 Carretera \\ Atlixco-Metepec, Puebla 74360, Mexico; dalramz0224@gmail.com (D.R.-R.); rosana.pelayo.c@gmail.com (R.P.) \\ * Correspondence: karlen@unam.mx; Tel.: +52-55-5622-9206 or +52-55-5622-9207
}

Citation: Mora-Roldan, G.A.; Ramirez-Ramirez, D.; Pelayo, R.; Gazarian, K. Assessment of the Hematopoietic Differentiation Potential of Human Pluripotent Stem Cells in 2D and 3D Culture Systems. Cells 2021, 10, 2858. https://doi.org/ $10.3390 /$ cells10112858

Academic Editor: Andre Larochelle

Received: 30 August 2021

Accepted: 8 October 2021

Published: 23 October 2021

Publisher's Note: MDPI stays neutral with regard to jurisdictional claims in published maps and institutional affiliations.

Copyright: (c) 2021 by the authors. Licensee MDPI, Basel, Switzerland. This article is an open access article distributed under the terms and conditions of the Creative Commons Attribution (CC BY) license (https:/ / creativecommons.org/licenses/by/ $4.0 /)$.

\begin{abstract}
Background. In vitro methods for hematopoietic differentiation of human pluripotent stem cells (hPSC) are a matter of priority for the in-depth research into the mechanisms of early embryogenesis. So-far, published results regarding the generation of hematopoietic cells come from studies using either 2D or 3D culture formats, hence, it is difficult to discern their particular contribution to the development of the concept of a unique in vitro model in close resemblance to in vivo hematopoiesis. Aim of the study. To assess using the same culture conditions and the same time course, the potential of each of these two formats to support differentiation of human pluripotent stem cells to primitive hematopoiesis without exogenous activation of Wnt signaling. Methods. We used in parallel 2D and 3D formats, the same culture environment and assay methods (flow cytometry, IF, qPCR) to investigate stages of commitment and specification of mesodermal, and hemogenic endothelial cells to CD34 hematopoietic cells and evaluated their clonogenic capacity in a CFU system. Results. We show an adequate formation of mesoderm, an efficient commitment to hemogenic endothelium, a higher number of CD34 hematopoietic cells, and colony-forming capacity potential only in the 3D format-supported differentiation. Conclusions. This study shows that the $3 \mathrm{D}$ but not the $2 \mathrm{D}$ format ensures the induction and realization by endogenous mechanisms of human pluripotent stem cells' intrinsic differentiation program to primitive hematopoietic cells. We propose that the 3D format provides an adequate level of upregulation of the endogenous Wnt/ $\beta$-catenin signaling.
\end{abstract}

Keywords: hPSC; primitive hematopoietic differentiation; Wnt signaling; embryoid body; 3D culture

\section{Introduction}

In vitro generation of human hematopoietic stem cells from embryonic and induced pluripotent stem cells (hPSC) serves multiple beneficial purposes: mechanistic studies of hematopoiesis, development of cell therapy for hematological diseases, induced transplant tolerance, disease modeling, and drug screening, among others [1] [2]. The current protocols for the generation of hematopoietic cells are based on the recapitulation of developmental signals similar to those in the embryo. In vitro, hematopoietic progenitors originate from mesoderm through hPSC differentiation, as a part of the process that starts upon the exposure of hPSCs to the appropriate dose of bone morphogenic factor 4 (BMP4) [3]. Then the mesoderm begins to form the hemogenic endothelium resulting in hematopoietic commitment (Figure 1) [4,5]. Much effort has been applied to defining the appropriate differentiation conditions required to obtain hematopoietic stem cells in vitro; for example signal gradients [6], cytokines in suitable combinations with the appropriate timing and specific microenvironments, such as feeder cells or artificial niches and with different culture systems, such as embryoid bodies (EBs) [7]. The EB has been employed widely in studies 
of hematopoiesis to parallel the course of embryonic development and to investigate the primitive (and definitive) hematopoiesis represented by the hemangioblast [8-10] that produces erythroid cells and monocytes [4,5,11-13]. Alongside, a 2D monolayer has been considered to be adequate for the induction of primitive hematopoiesis [14-18]. In both EB and 2D Monolayer systems, exogenous Wnt/catenin pathway stimulation was used to enhance the definitive developmental compartmentalization/wave of hematopoiesis. Together, these studies contributed to the current concept of in vitro hematopoietic differentiation and raised questions regarding the reasons for using either EBs or monolayers to produce hematopoietic cells (See Table 1). Recent studies demonstrated the advantages of 3D organoid protocols of hPSC differentiation and generation of clinical-grade definitive human progenitor cells and stem cells of hematopoietic lineages, potentially engraftable lymphoid progeny for transplantation studies and, erythroid progeny capable of producing adult hemoglobin [19]. 3D culture technology has also been improved by the employment of biomaterials to create 3D tumor models for drug screening $[20,21]$. Additionally, when applied in ectodermal-derived lineages, the 3D format improved neuronal differentiation, and the formation of a neural network, recapitulating brain tissue-like environments suitable in a disease model-specific extracellular aggregation [22]. In the past, the comparative potentials of $2 \mathrm{D}$ and $3 \mathrm{D}$ culture platforms for supporting in vitro hematopoietic differentiation of hPSCs have been explored, to improve their pluripotency and escalate cell productivity $[23,24]$.

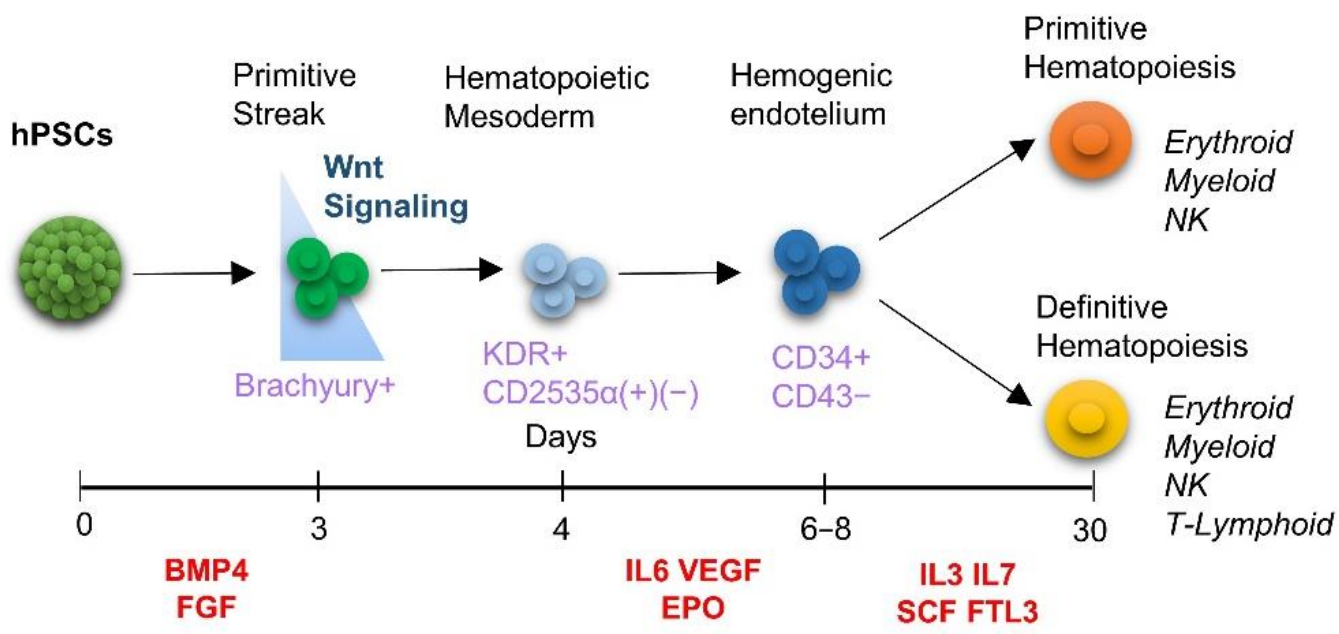

Figure 1. Schematic representation of current knowledge of in vitro differentiation of human pluripotent stem cells to hematopoietic lineage. Hematopoietic differentiation of hPSCs is depicted based on the protocols in the literature. Hematopoietic differentiation occurs in a consecutive stage-specific manner, starting with the BMP4-induced mesodermal commitment (days $0-4$ ) followed by conventionally employed exogenous activation of Wnt/ $\beta$-catenin signaling at this initial stage. The Lateral plate (Day 3-4) mesoderm generates endothelial progenitors (4-6), hemogenic endothelium with the potential to hematopoietic lineages (Day 6-8). At this latter stage, the population splits into two developmental programs/waves: primitive hematopoiesis, characterized by the formation of monocytic cell types, and definitive hematopoiesis with lymphoid potential. Abbreviations: FGF: Fibroblast Grow Factor, IL3: Interleukin 3 IL6: Interleukin 6, IL7: Interleukin 7, VEGF: Vascular Endothelial Grow Factor, EPO: Erythropoietin, SCF: Stem Cell Factor, FLT3: receptor-type tyrosine-protein kinase. 
Table 1. Selected publications on the independent use of 3D/Embryoid Body and 2D/monolayer systems in hematopoietic differentiation from hPSCs. The table displays the differences in the generation of hematopoietic lineages under these two conditions. Additionally, results show the importance of Wnt/ $\beta$-catenin signaling activation during in vitro differentiation of hPSCs of the definitive hematopoietic fate.

\begin{tabular}{|c|c|c|c|c|c|c|}
\hline Study & hPSCs & $\begin{array}{l}\text { Culture } \\
\text { System }\end{array}$ & $\begin{array}{c}\text { Day(s) } \\
\text { Hematopoietic } \\
\text { Commitment }\end{array}$ & $\begin{array}{l}\text { Hematopoietic } \\
\text { Wave }\end{array}$ & Lineage Cells & $\begin{array}{c}\text { Wnt } \\
\text { Activation }\end{array}$ \\
\hline $\begin{array}{l}\text { Yanagimachi } \\
\text { et al., } 2013\end{array}$ & hESCs, hiPCSs & 2D/Monolayer & 6 & Primitive & $\begin{array}{l}\text { Monocytes, Dendritic } \\
\text { Cells }\end{array}$ & No \\
\hline $\begin{array}{l}\text { Sturgeon et al., } \\
2014\end{array}$ & $\begin{array}{l}\text { hESCs, } \\
\text { HiPSCs }\end{array}$ & 3D/EB & 6 & $\begin{array}{l}\text { Primitive } \\
\text { definitive }\end{array}$ & $\begin{array}{c}\text { Monocytes, } \\
\text { Lymphoid Lineages }\end{array}$ & Yes \\
\hline Ruiz et al., 2019 & hiPCSs & 2D/Monolayer & $5-9$ & Primitive & $\begin{array}{c}\text { Primitive } \\
\text { Hematopoietic Cells }\end{array}$ & Yes \\
\hline $\begin{array}{c}\text { Niwa et al., } \\
2011\end{array}$ & hiPSCs & 2D/Monolayer & 6 & Primitive & Monocytic Lineages & No \\
\hline $\begin{array}{l}\text { Kennedy et al., } \\
2007\end{array}$ & hESCs & 3D/EB & $5-6$ & Primitive & $\begin{array}{c}\text { Early Hematopoietic } \\
\text { Progenitors }\end{array}$ & No \\
\hline $\begin{array}{c}\text { Galat et al., } \\
2017\end{array}$ & hiPSCs & 2D/Monolayer & 5 & Primitive & Monocytic lineages & Yes \\
\hline $\begin{array}{c}\text { Ditadi \& } \\
\text { Sturgeon, } 2015\end{array}$ & hESCs & $3 \mathrm{D} / \mathrm{EB}$ & $8-15$ & $\begin{array}{l}\text { Primitive, } \\
\text { Definitive } \\
\text { progenitors }\end{array}$ & $\begin{array}{c}\text { Hemogenic } \\
\text { Endothelium and } \\
\text { early hematopoietic } \\
\text { progenitors }\end{array}$ & Yes \\
\hline $\begin{array}{c}\text { Knorr et al., } \\
2013\end{array}$ & hIPSC, hESCs & $3 \mathrm{D} / \mathrm{EB}$ & 11 & Definitive & Nk, Lymhpoid & No \\
\hline
\end{tabular}

In the same context, comparison between 2D and 3D conditions has been performed in studies of other differentiation models, such as osteogenic differentiation [25] and, widely in cardiac differentiation. Studies with hPSC-derived cardiomyocytes found that the differentiation potential was affected by the choice of culture platforms, either it is $2 \mathrm{D}$ or 3D [26-30]. This study aimed to reveal differences between the 2D and 3D conditions observable during the generation of primitive hematopoietic cells from hPSCs in the same media and cytokines. Previously, some differences between 2D and 3D settings in lineage specification processes, as well as its (3D) advantages, have been noted in independent studies [14,31-33]. However, in independent experiments, due to the extreme plasticity of the early embryogenesis, the microenvironment differences can influence distinctly the developmental processes hence only by the use of the same experimental setting, same culture condition, parallel assays, and principles of interpretation can we highlight reliably, the intrinsic differences between the two culture methods observed at each of the steps of the in vitro induction and differentiation. To address this issue, we have undertaken comparative experiments of $2 \mathrm{D}$ and $3 \mathrm{D}$ systems regarding the capacity of each of them to support the primitive hematopoietic differentiation using a commercial embryonic stem cell line and an induced pluripotent stem cell line generated in our lab (Figure S1A,B).

\section{Materials and Methods}

\subsection{Embryonic Stem Cell and iPS Cell Lines}

The ES cell line ESI-017 was purchased from ESI-BIO (Alameda, CA, USA). Induced Pluripotent Stem Cells were generated as described previously [34]. Briefly, human dermal fibroblasts (ATCC catalog number PCS-201-010) were transduced with lentiviral vectors containing the genes encoding reprogramming factors Oct 4, Sox2, Nanog, Klf4 and, C-Myc resulting in the formation of colonies on day 15 to 20 days post-infection. Colonies were then selected according to morphology and compaction and expanded to passage 30 and maintained until ready for differentiation. The use of human cells in this study has been 
approved by the Ethics Committee of the Biomedical Research Institute of the National Autonomous University of Mexico (UNAM).

\subsection{Cell Culture under 2D/Monolayer and 3D/Embryoid Body Conditions}

Human ES and iPS cell colonies were cultured as 2D/monolayer and 3D/EB in Essential 8 medium, E8 (Gibco; Thermo Fisher Scientific, Inc., Waltham, MA, USA, then detached using $0.1 \%$ Type IV Collagenase (Sigma-Aldrich, St. Louis, MI, USA) followed by mechanical disaggregation. The $2 \mathrm{D} /$ monolayer cells were seeded at a density of $1 \times 10^{4}$ onto Matrigel () (MG)-coated plates (Corning, NY, USA) whereas for 3D culture untreated, ultralow attachment plates (Nunc, Rochester, New York, USA) were used. Cell clumps were maintained in E8 medium until spheres were grown to no more than $300 \mu \mathrm{M}$ in diameter. Obtained spheres were passaged after collagenase treatment and mechanical disaggregation. Cell clumps of around 100 to $200 \mu \mathrm{m}$ were seeded and cultured in the same conditions until initiation of differentiation (day 0 ).

\subsection{Mesodermal, Hematoendothelial and Hematopoietic Differentiation}

To observe the effects of the two (2D and 3D) culture systems on differentiation, neither Wnt pathway activators nor inhibitors were added to the media as conventionally done (See Table 1). The differentiation of the hPSCs took place in 12-well plates (Nunc) treated with MG for the 2D and in ultra-low attachment non-treated plates for 3D. Mesodermal differentiation proceeded in Stempro 34 (Sigma-Aldrich) medium supplemented with $10 \times$ ITS (Gibco), 40 ng/mL BMP4, 10 ng/mL FGF2 (Preprotech), $50 \mu \mathrm{g} / \mathrm{mL}$ Ascorbic Acid (Sigma-Aldrich), 100× Glutamax (Gibco) and Gentamicin (Gibco) from day 0 to day 4 . On day 4 , the medium was changed to Stempro 34 with $10 \times$ ITS, $100 \times$ Glutamax, Gentamicin, $10 \mathrm{ng} / \mathrm{mL}$ FGF, $10 \mathrm{ng} / \mathrm{mL}$ VEGF; $10 \mathrm{ng} / \mathrm{mL}$ of IL6 and $2 \mathrm{U} / \mathrm{mL}$ EPO (Preprotech) for three more days (days 4 to 6). On day 6 the medium was replaced with hematopoietic differentiation Stempro 34 medium containing the above supplements plus $50 \mathrm{ng} / \mathrm{mL} \mathrm{SCF}$, $5 \mathrm{ng} / \mathrm{mL}$ IL7, $5 \mathrm{ng} / \mathrm{mL}$ Flt3 and $10 \mathrm{ng} / \mathrm{mL}$ IL3 (Preprotech Rocky Hill, USA). CHIR99021 $(3 \mu \mathrm{M})$ and IWR-1(1 $\mu \mathrm{M})$ (Sigma), were added only when indicated.

\subsection{Hematopoietic Colony Assay}

Analysis of hematopoietic colony formation potential of the hematopoietic stem cells was performed by plating $2 \times 100$ cells in Methocult (Stem Cell Technologies Inc. Vancouver, Canadá) supplemented with SCF (100 ng/mL), EPO (2 U/mL), IL-6 (5 ng/mL), IL-3 (40 ng/mL), TPO (40 ng/mL) (Preprotech). Colonies were quantified after 30 days. Cells were separated from the Methocult by centrifugation and fixed for Wright staining.

\subsection{AFT024 Co-Culture for Further Hematopoietic Differentiation}

To generate further hematopoietic lineages, we employed co-culture with the AFT024 cell line, which is derived from mouse fetal liver stroma, this cell line expresses DL1, an important factor during the acquisition of advanced hematopoietic fates. Cells were purchased from ATCC (SCRC-1007) and transduced with a lentiviral GFP reporter (pLenti CMV GFP Puro, Addgene) [34]. Passage 4 cells were expanded with Optimem (Gibco) and $5 \%$ FBS (Gibco) until confluency and then seeded at 10,000 cells per well in 96 well plates. On days 6 to 8,2D/monolayer and 3D/EB cells were plated on the AFT024 monolayers with Optimem medium supplemented with 1\%FBS, 100X Glutamax, and IL7, IL3, SCF with a change of monolayer every 5 days during 30 days [35].

\subsection{Flow Cytometry Analysis}

Flow cytometry analysis was done in a FACSCanto cytometer (Becton Dickinson, NJs, USA). Fluorophore-conjugated antibodies used for flow cytometry immunostaining are listed in Supplementary Table S2. Immunostaining was performed as recommended in the technical datasheet for each antibody. Analysis of flow cytometry acquisitions was performed using FACSdiva Software (Becton Dickinson, BD Biosciences, CA, USA) 
and FlowJo v10.0.7 (FlowJo, LLC, BD Biosciences, CA, USA). Ten thousand events were recorded for each sample. Analysis of Flow Cytometry Data and the fold increase in mean fluorescence was performed with FlowJo v10.0.7.

\subsection{Immunofluorescence}

Cells were seeded on MG-coated chamber slides (Nunc-Labtek, Thermo Scientific, USA). Cells were fixed with $4 \%$ PFA for $15 \mathrm{~min}$ at room temperature (RT) and then permeabilized with $0.2 \%$ Triton in PBS for 10 min. Cultures were blocked using 1\% BSA plus $0.2 \%$ Triton in PBS for $30 \mathrm{~min}$ at RT and then incubated with primary antibody in blocking solution for $1 \mathrm{~h}$ at RT or overnight at $4{ }^{\circ} \mathrm{C}$. After washing, the secondary antibodies were added, washed and cells were put in blocking solution for $1 \mathrm{~h}$ at RT in the dark. After washing, nuclei were stained with Hoechst 33342 (Invitrogen, CA, USA). Epifluorescence analysis was performed using an Olympus IX71 phase-contrast fluorescence microscope and images were analyzed with QCapture Suite software (QImaging, Surry, BC, Canada). Microscopy analysis was performed using a Nikon confocal microscope and images were analyzed with the Image J (ImageJ, U. S. National Institutes of Health, Bethesda, Maryland, USA) software, Z Stacks of the 3D cultures were obtained using the Bioformats plugin. The list of antibodies used and the catalog number is available in Supplementary Table S2.

\subsection{Gene Expression Analysis by RT-qPCR}

Total RNA was isolated using TRIzol reagent according to the manufacturer's instructions (Invitrogen, CA, USA) followed by DNase I (Invitrogen, Waltham, MA, USA) treatment and an RNA clean-up using the RNeasy mini kit (Qiagen, Hilden, Germany). Reverse-transcription reactions were performed using a One-step RT-PCR kit (Qiagen). Quantitative RT-PCR (RT-qPCR) reactions were performed using KAPA SYBR FAST (Roche) master mix on a Rotor-Gene 6000 thermal cycler (Qiagen). Data analysis was performed using Rotor-Gene Q series Software (Qiagen) and relative expression was calculated using Pfaffl's efficiency calibrated method [36]. The primers for the PCR experiments are listed in Supplementary Table S1.

\subsection{Statistical Analysis}

Values are given as mean with SE, and statistical significance was performed from at least three independent biological replicates. Student's $t$-test or analysis of variance (ANOVA) were performed to analyze two or more groups of means, respectively, using GraphPad Prism version 6.04 for Windows, (GraphPad Software, La Jolla, CA, USA). Tukey's post hoc test was performed to determine the statistical significance of the ANOVA.

\section{Results}

\subsection{D/EB and 2D/Monolayer Culture Conditions Differ in Their Capability to Produce Hematopoietic Cells}

Our main objective was to compare the capacity of these formats to support the cell commitment events during differentiation of hPSC to primitive hematopoiesis in the lack of exogenous stimulation of Wnt/ $\beta$-catenin shown previously to direct differentiation to definitive hematopoietic lineages [37-42]. To this purpose, we performed simultaneous differentiation in these formats, with the same culture environment and assay methods, without the addition of any Wnt/ $\beta$-catenin exogenous stimulator like CHIR99021. Figure 2A depicts the cells' morphological changes in 2D and 3D formats on days $0,4,6$, and 8 of hPSCs differentiation driven exclusively by endogenous factors. Before differentiation, we tested the capacity of the protocol we used to ensure similar expanding and pluripotency properties of the passage $30 \mathrm{hESC}$ and hiPSC, by seeding them in the same media as a monolayer at a density of $1 \times 10^{4}(2 \mathrm{D})$ or as spheres (3D) (see the section of Materials and Methods) and culturing for 5 days. The Immunofluorescence analysis with antibodies to Tra160, Oct4 and NANOG showed no significant differences between the two cultures, evidencing that hPSCs used in the study were similar from the 
start of the differentiation, hence the differences that could arise will be from the specific effects of the culture formats (Figure 2B) (Figure S1C).

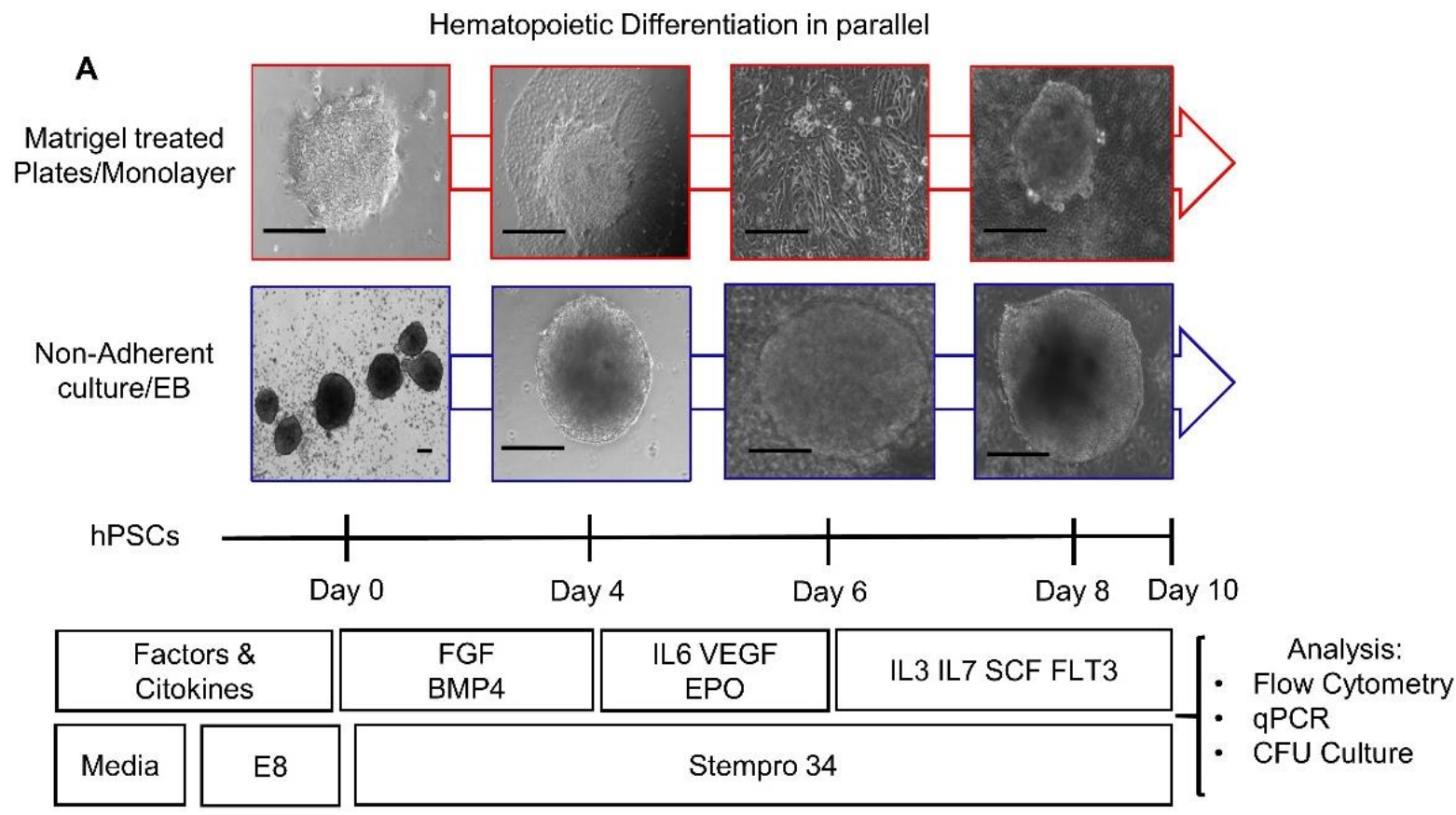

B

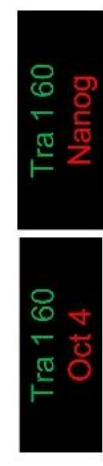

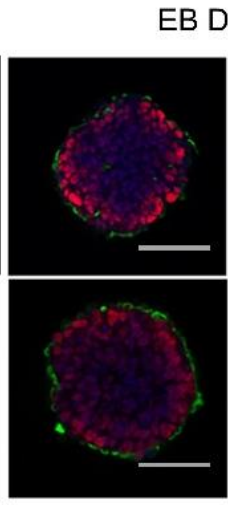

EB Day 0

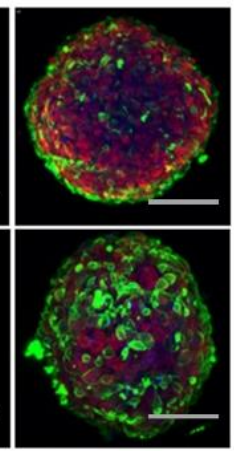

Mono Day 0
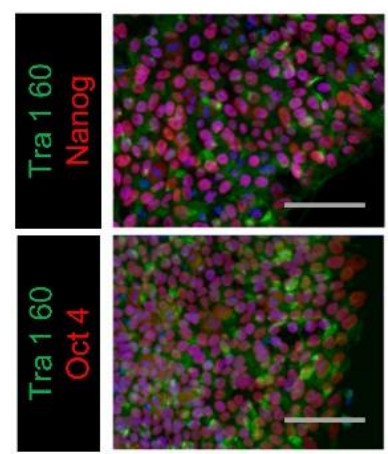

Figure 2. Comparison of the pluripotency of hPSCs in 2D/monolayer and 3D/EB culture systems. (A) Experimental procedure of in vitro differentiation in parallel settings showing representative phase-contrast images of cells at Day 0 , Day 4, Day 6 and Day 8. (B) Immunofluorescence analysis of 3D/EBs clones and their cross-sections, and 2D/Monolayer cells, stained by antibodies to Tra-160, Oct4 and NANOG. Scale Bars: EB: $100 \mu \mathrm{M}$, Monolayer: $100 \mu \mathrm{M}$.

As the pluripotency of hPSCs was similar in both 3D and 2D colonies, we set on to find possible differences in the hematopoietic commitment of the cells under the two culture conditions. As was reported (see references in Table 1), early hematopoietic commitment can be identified starting from Day 6 through detection and quantification of a cell cohort with the CD34+/(Kinase domain receptor), KDR+ phenotype. Although CD43 would be adequate for the identification of hematopoietic populations in these experiments, yet we found the CD34+/KDR+ co-expression preferable to identify cells in transition from mesoderm to hematopoietic and endothelial phenotypes. With this intention in mind, we measured the number of $\mathrm{CD} 34+/ \mathrm{KDR}+$ cells by flow cytometry and compared the numbers of CD34-positive cells under the two culture formats on days 4, 6, and 8 (Figure 3A) (Figures S2-S4). A higher number of CD34+/KDR+ cells in the 3D/EB condition was 
found. Additionally, we measured the colony-forming capacity of cells from both systems grown on methylcellulose for 30 days (Day $6+24$ ). For this purpose, day 6 cells were divided in half, one of which was used for FACS analysis and the other half used in the methylcellulose colony formation experiments. (Figure 3B). Two hundred cells $(n=3)$ from each of the formats were used and obtained on average seven colonies (four of them are shown in Figure 3). Although CFU's were already detectable at 10-15 days, we continued to culture up to 30 days $(6 \mathrm{~d}+24 \mathrm{~d})$, cells were isolated and then wright-stained and identified as CFU-GM colonies. Clear differences between the two culture formats were revealed: round and refractive groups of cells were found in the 3D/EB while monolayer/2D cultures did not show colony-formation (Figure 3B). We did not separate and identify more CFU types, considering that only one type already demonstrated a sharp difference between the two formats. As there were no colonies at the $2 \mathrm{D} /$ monolayer condition $(n=3)$, the quantitative determination of CFU capacities was not performed.

The cytometry analysis results described in Figure 3B showed that only the suspension (3D/EB) culture format produced a population of CD34+KDR+ cells. When further analyzing this double-positive subset, we found that this cell population expresses almost in its entirety CD31 (97.5\%). This suggested the emergence of a hemogenic endothelial population $(\mathrm{CD} 34+\mathrm{KDR}+\mathrm{CD} 31+)$. Simultaneously, we found evidence (lower panel) of a population that is positive for CD34, and positive for CD31, but negative for KDR, a possible indication of non-hematopoietic endothelial cells, showing that hematopoietic and non-hematopoietic cells can arise simultaneously during this period of differentiation (44-47). This finding correlates with the formation of colonies in the 3D and the lack of colonies in the monolayer format. Immunofluorescence showed on day 6 the coexpression of CD34, KDR, and CD43 in the 3D but not in the 2D format (Figure 3C). These results indicated that the $2 \mathrm{D} /$ monolayer system was significantly inferior in supporting the hematopoietic differentiation, basically, and specifically, in the development of clonogenic CD34 hematopoietic cells. Additionally, to know whether the observed distinctions were due to a slower differentiation of 2D/Monolayer cells, we compared the time course of differentiation of the cells in the two formats at later time points (Day 8 and Day 10) (Figure 3A). The results showed a consistent time course pattern of CD34+ cell generation on days 8 and 10 (Figures S4 and S5), indicating that there was not a higher number of positive cells at any time point during the differentiation of the 2D/Monolayer. Once the above differences between the two systems were observed, we sought to find out the earliest time point at which these differences became apparent. To this end, we performed qRT-PCR analysis of the expression of day 4 cells of key mesodermal commitment markers: Brachyury (T), BMP, KDR, Plate derived growth factor receptor (PDGFR), and Sox17, a marker associated with endodermal lineage but also known as a differentiation-related factor during the acquisition of endothelial phenotype required for the early hematopoietic fate establishment [43-46]. We found that the mesoderm was phenotypically well established in 3D/EB by day 4; in contrast, the 2D format cells showed a reduced or no commitment (Figure 3D) (see the primers in Supplementary Table S1). In particular, the expression of Brachyury ( $\mathrm{T}$ ) was two-fold higher in 3D/EB system than in the 2D system $(p \leq 0.001)$. We analyzed the expression of the KDR gene, an important mesodermal marker that, unlike the $T$ gene, remains active in cells undergoing hematopoietic commitment [45]. In our experiments, the 3D/EB mesoderm cells showed a high expression of KDR, whereas the $2 \mathrm{D} /$ monolayer mesoderm cells were almost negative for this marker, suggesting that none or very few of these cells were competent for hematopoiesis. PDGFR has been used to identify lateral plate mesoderm and to distinguish it from paraxial mesoderm [47,48]. We found that the level of expression of the PDGFR gene by $3 \mathrm{D} / \mathrm{EB}$ cells exceeded more than twofold its expression by the $2 \mathrm{D} /$ monolayer cells $(p \leq 0.001)$, suggesting that the $2 \mathrm{D}$ system had a weaker capacity of producing lateral mesoderm. The expression patterns of all these markers suggested that the $2 \mathrm{D} /$ monolayer system was deficient for the appropriate induction in the mesodermal cells (positive for T and KDR) of commitment to the hematopoietic lineage (KDR), displaying, at the same time, a strong selectivity for lateral 
mesoderm (PDGFR) commitment to early (primitive) hematopoiesis (So $\times 17)$. Figure 3E compares T and KDR immunofluorescence staining on day 4 . Additionally, in line with the above results (Figure 3D), we found that the mesodermal marker Brachyury (T) was expressed by cells of both formats, but to a different extent. Commitment was distinctively achieved in the two systems. However, as there was little or no KDR staining in 2D cells, it can be posited that 2D/Monolayer condition could support a partial mesodermal commitment but is still insufficient to fully achieve hematopoietic specification. In common, these results suggested a weaker capacity to induce lateral mesoderm of the 2D system, thus resulting in a diminished hematopoietic commitment.

A

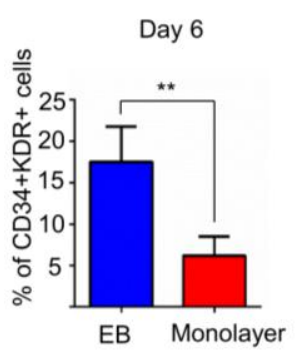

B

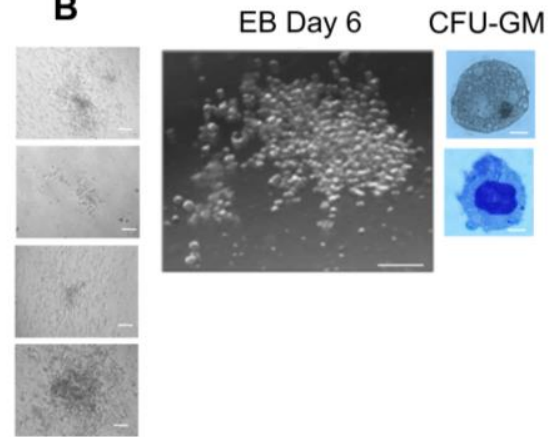

C

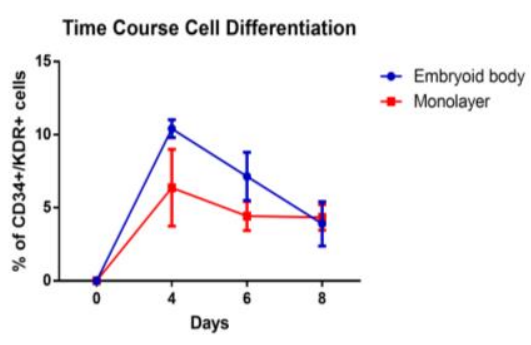

Days

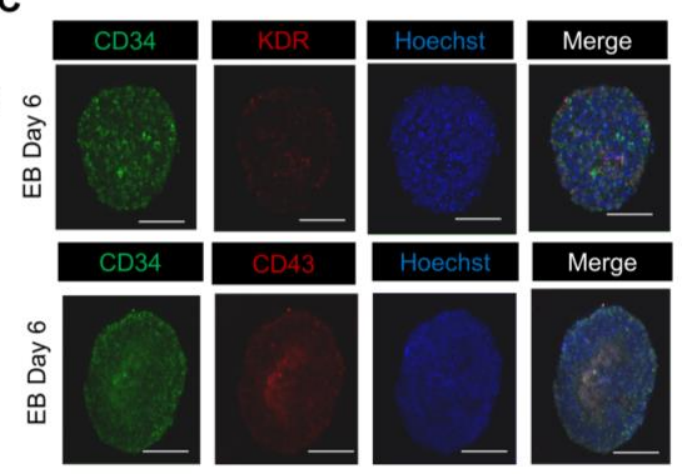

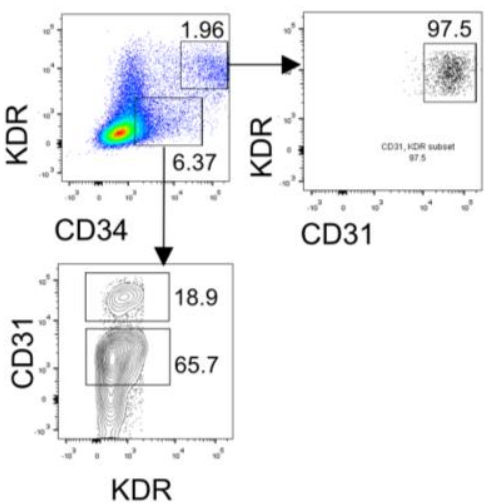

KDR
Monolayer Day 6

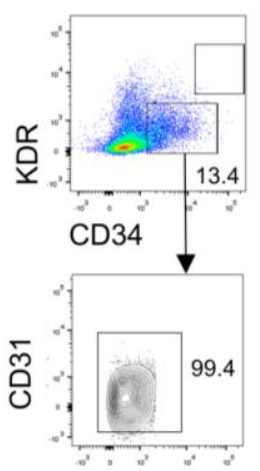

KDR

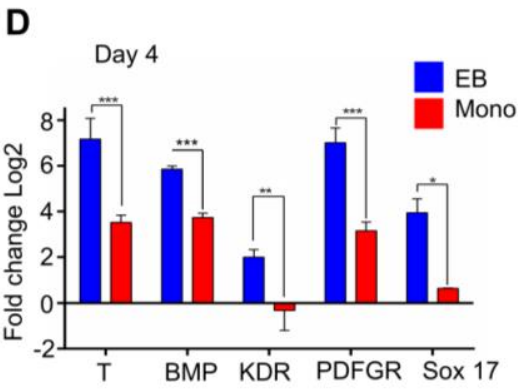

Monolayer Day 6

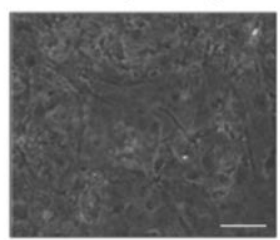

E
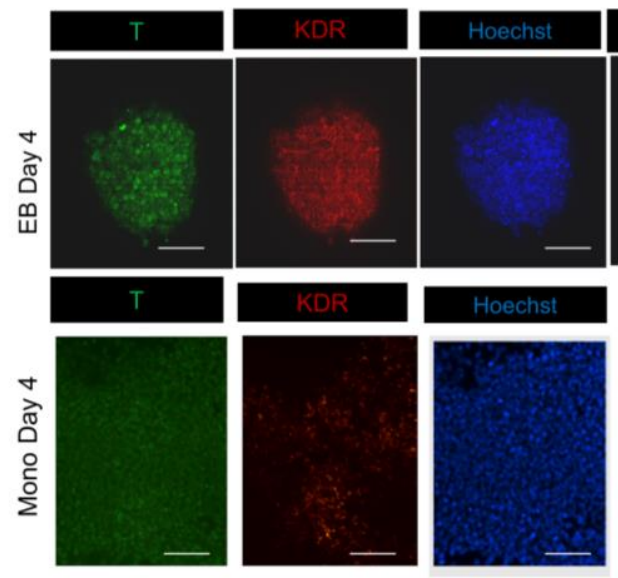

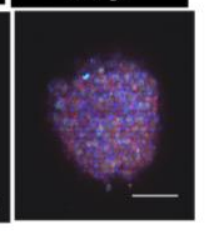

Merge

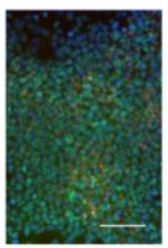

Figure 3. 3D/EB suspension and 2D/monolayer formats differ significantly in their capability of producing hematopoietic cells from hPSCs. (A) Comparison of the percentages of the cells with CD34+/KDR phenotype generated by the 3D and $2 \mathrm{D}$ systems on day 6 (Mean $\pm \mathrm{SEM}) n=4,{ }^{* *} p \leq 0.01$. Time course of CD34/KDR cells in 3D and 2D formats (B) Phase contrast image of hematopoietic colonies in methylcellulose shown for 3D/EB on day 30 after Wright-Staining (see methods), showing images of a typical macrophage and a monocyte. No such cells were present in the 2D system. Flow cytometry 
analysis of the day $63 \mathrm{D} / \mathrm{EB}$ and 2D/monolayer cells for co-expression of CD34 and KDR (without sorting of CD34 cells) rectangles indicate the populations of interest with antibody stain. Abbreviations: CD34: Cluster of differentiation 34, CFU-GM: Colony-forming unit Granulocyte-Macrophage. (C) Immunofluorescence of hematopoietic markers CD34, KDR and CD43 on Day 6 of the 3D/EB cells; images were processed with Image J to obtain sections (z-stack) of EB using the bio formats plug-in (see methods). Abbreviations: T: Brachyury. (D) Quantitative RT-PCR determined expression levels of mesodermal genes on day 4 ; bars show the expression levels of the genes relative to the GAPDH gene; (Mean \pm SEM) $n=3$, ${ }^{*} p \leq 0.05,{ }^{* *} p \leq 0.01,{ }^{* * *} p \leq 0.001$. Results of three independent experiments with RNAs of iPS and ES cells are shown. (E) Immunofluorescence of mesodermal markers T and KDR on day 4 for 3D/EB and 2D/Monolayer at 20×, scale bar: $100 \mu \mathrm{m}$.

\subsection{Endogenous Wnt/Catenin Signaling Is Active in the 3D/EB System but Not in 2D/Monolayer System}

Since the 3D/EB culture system displays an enhanced mesodermal and hematopoietic commitment, we sought the possible causes of this phenomenon. Our group has previously reported that Wnt $\beta$-catenin is an essential regulator of the biphasic human somatic cell reprogramming [34]. In particular, Wnt activates mesendodermal genes during colony maturation towards the pluripotent state. Furthermore, in many studies $[17,41,49]$ exogenous Wnt/catenin was used to stimulate the production of hematopoietic populations from hPSCs. The comparative cytometry assays revealed that $\beta$-catenin was abundantly expressed by the 3D/EB and was almost non-expressed by the $2 \mathrm{D} /$ monolayer cells (Figure 4A) (Figure S6). In concordance with this higher presence of $\beta$-catenin in $3 \mathrm{D}$ cells, Figure 4B shows the higher expression in these cells of the Axin 2 gene, one of the Wnt transcription factors. Figure $4 \mathrm{C}$ shows immunostaining of the markers $\mathrm{T}$ and $\beta$-Catenin, it is observed that in the $3 \mathrm{D}$ condition both markers are present, however, in the monolayer condition, only $\mathrm{T}$ was present. These findings suggested that the high level of hematopoietic commitment in mesodermal cells and a more efficient differentiation to CD34+ cells with clonogenic potential in the 3D/EB format might be due to the higher endogenous levels of $\mathrm{Wnt} /$ catenin signaling. In line with this suggestion, the $2 \mathrm{D} /$ monolayer system's inability to sufficiently activate endogenous Wnt/catenin signaling might be a cause of its relative inefficiency to induce the commitment to the hematopoietic fate. To complement these data, we examined the two culture platforms for the ability to generate cells with advanced hematopoietic commitment; to this end, we sought to obtain lymphoid lineages by co-culturing the cells with AFT024 stromal cells as a feeder, providing in vivolike microenvironment for advanced hematopoietic differentiation. Figures S9 and S10 compare the two culture formats regarding the presence of CD56, which is most stringently associated with, but certainly not limited to, natural killer cells. As expected, the 3D format produced a higher number CD56 -positive cells than did the monolayer format on day 30 of culture.

Once it was shown that the 3D/EB system provides higher endogenous Wnt activity, we tested the effects of adding CHIR99021, a Wnt pathway activator, or IWR-1, a Wnt pathway inhibitor (Figures S7 and S8). The rationale for this was to look at the effects on hematopoietic differentiation of additional (to the endogenous) level of Wnt/ $\beta$-catenin induced by CHIR99022 in the 3D/EB cells (Figure 4D). It is known [50] that endothelial cells (ECs) are generated from mesoderm during hPSC differentiation, and are comprised of two sub-populations: non-hemogenic (vascular) and hemogenic endothelium; it is also known that the CD34+/CD31+ population is a hemogenic endothelium with primitive potential [51]. As was described above, we split the EB+CHIR derived cells into two portions, one of which was cultured in CFC and the other one subjected to FACS. We found that the 3D cells acquired an advanced clonogenic capacity evidenced by the generation of erythroid colonies (CFU-E) (Figure 4D) (Figure S11). In Figure 4E, we show that activation of Wnt by CHIR99021 depleted the CD31+ subpopulation that was present in the 3D/EB condition without Wnt activation. Additionally, activation of the Wnt pathway depleted the CD31+/KDR+ fraction that forms a part of the endothelial- primitive hematopoietic lineage cells (Figure 4E). Our results on the depletion of these populations correlate with 
the published (Table 1) data, indicating that exogenous Wnt activation is a means to launch both primitive and definitive hematopoietic programs.

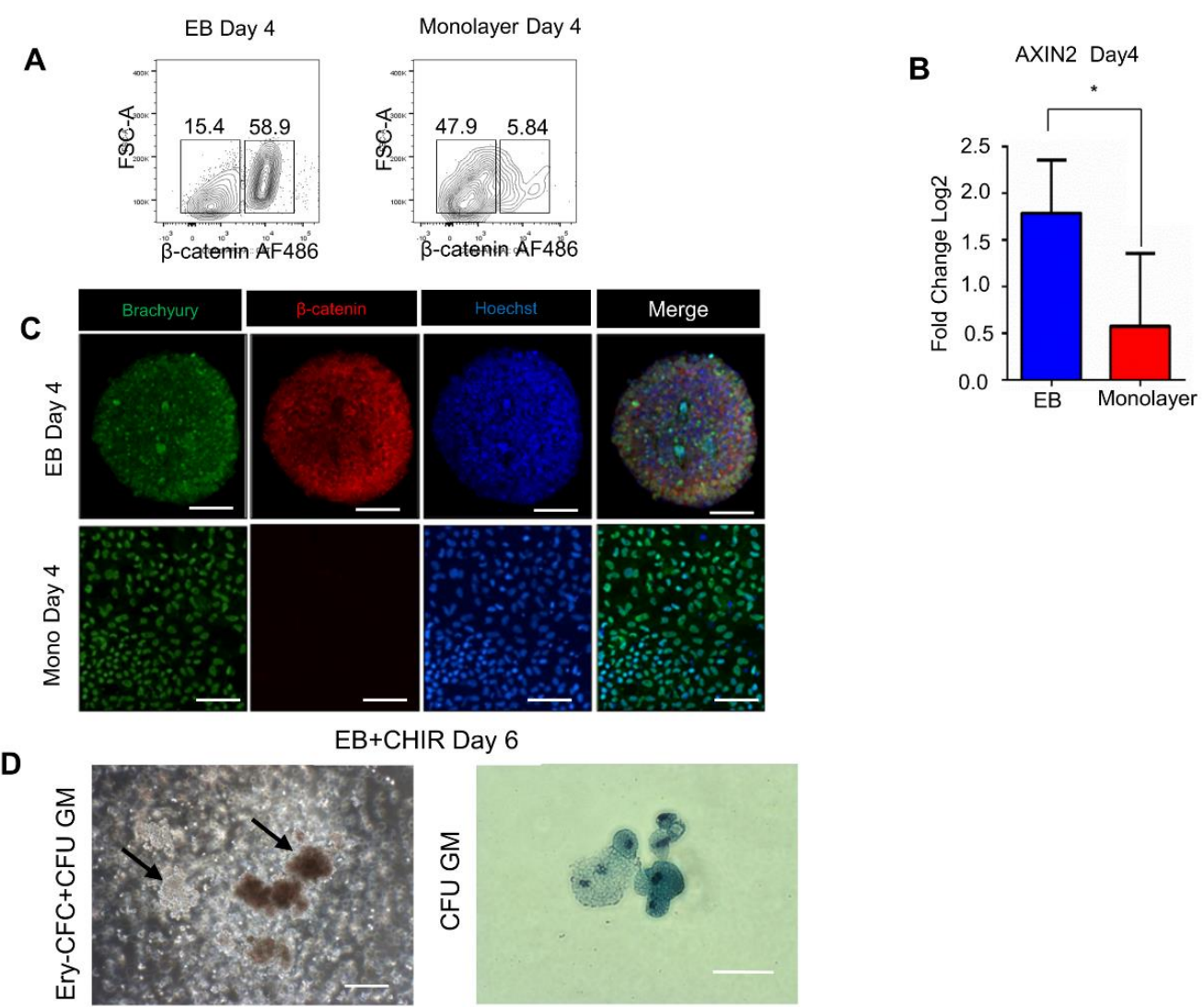

E

Day 6
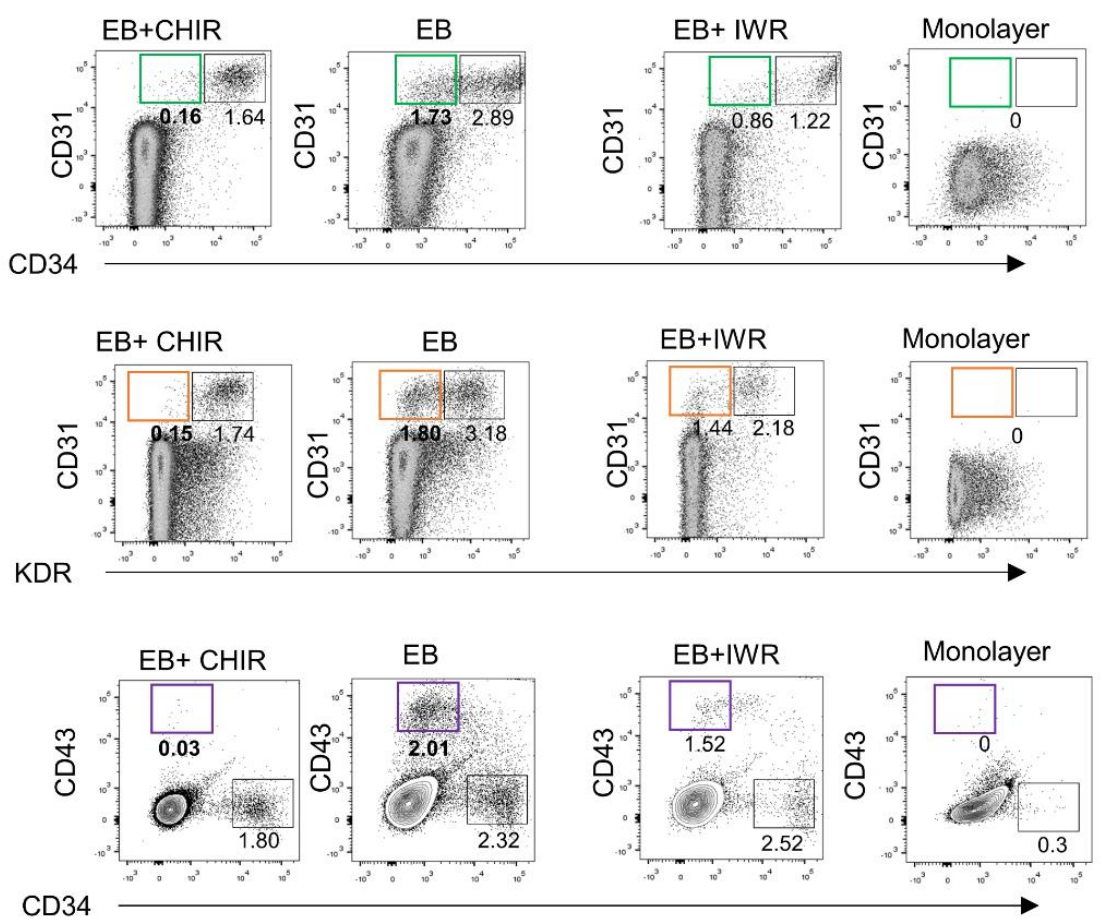

Figure 4. Endogenous activity of Wnt/ $\beta$-catenin signaling in 3D/EB and 2D/Monolayer cells on day 4 of PSC differentiation. (A) Representative flow cytometry analysis of Day 4 cells, comparison of $\beta$-catenin-positive cells determined by FACS, and 
(B) Quantitative PCR analysis of Axin2, and (C) Immunofluorescence of Brachyury and $\beta$-catenin. Scale Bars: $100 \mu \mathrm{m}$, $($ Mean $\pm \mathrm{SEM}) n=3,{ }^{*} p \leq 0.05$. (D) Phase-contrast images of disaggregated 3D/EB+CHIR day 6 cells cultured in methylcellulose; arrows indicate colonies of EryCFC and CFU-GM wright-stained. (E) Representative flow cytometry analysis of Day 63D/EB cells after the use of Wnt activator/inhibitor showing the results of the treatment either with CHIR or with IWR, green rectangle shows the population of interest, the endothelial component in CD31/CD34 cells, orange rectangle shows the endothelial component in the CD31/KDR cells and purple rectangle distinguishes the primitive hematopoietic component in the CD43/CD34 cells. Source of the cells: CD34/KDR populations (D), CD31/CD34, KDR/CD31, and CD34/CD43 populations.

Previously, Keller's and Slukvin's groups (see Table 1) demonstrated that the mesoderm gives rise to a hematopoietic CD34+/CD43- population detectable on day 6 of hPSC differentiation. This population expresses the markers of hemogenic endothelium and possesses multi-lineage (definitive) hematopoietic potential. On the other hand, the CD34+/CD43+ population may represent primitive hematopoietic cells. We found that when 3D/EB cells were treated with CHIR, the CD43+ fraction was absent in the day 6 cultures. We consider that our EB+CHIR Wnt activation condition depleted the primitive CD34+CD43+ in favor of the CD34+CD43- phenotype, in line with Keller's finding. Conversely, this population remained stable in untreated $3 \mathrm{D} / \mathrm{EB}$ cultures. Altogether, these results further support the notion of the 3D/EB condition's higher capacity to mimic the early embryonic events (primitive wave of hematopoiesis) during in vitro differentiation (Figure 4E). In contrast, the expression levels of the markers CD34, CD31, KDR, and CD43 were low in the EB+IWR cells, providing additional confirmation that the $2 \mathrm{D} /$ monolayer is by itself insufficient to generate hematopoietic cells (Figure 4D,E).

\section{Discussion}

In previous studies (e.g., the references in Table 1) with the 3D/EB culture format, it was shown that in the presence of growth factors, specific cytokines, and other ingredients, hPSCs exposed to appropriate doses of BMP4 [38] were committed through activation of T (Brachyury) and KDR genes to the mesoderm, then through hemogenic endothelium, to transient primitive hematopoietic bipotential hemangioblast cells capable of differentiating into hematopoietic and endothelial cells. On the other hand, in independent experiments using the 2D format, hPSCs required exogenous stimulation of Wnt signaling for the generation of definitive hematopoietic populations. The specific contribution of each of these two formats is difficult to evaluate in independent experiments, because the distinctions in the microenvironment factors, different assay conditions and differences in interpretation of results could obscure their effects on in vitro hematopoietic processes. This study compares the potentials of these two formats to support the hPSCs hematopoietic differentiation in the same experimental settings without exogenous stimulation of Wnt signaling. Our results describe in detail how each format supports hematopoietic differentiation, starting from the mesoderm formation and commitment to the posterior stages up to the clonogenicity stage. To our understanding, this is the first such study whose results contribute to the estimation of the potential of the endogenous machinery to realize the differentiation program in two experimental settings and use these results in the conceptualization of in vitro hematopoiesis. Our results support the view that the 3D/EB system efficiently mimics the in vivo embryonic events. In addition, our results provide a tentative explanation for the higher capacity of the 3D/EB system, by showing that endogenous Wnt/catenin signaling is more active in this format on day $4[39,41,49]$. The novelty in our findings is the demonstration of the importance of adequate activation of endogenous Wnt signaling by BMP in the 3D format, which is required for robust mesoderm formation through the activation of the genes encoding $\mathrm{T}$ and KDR, which determines successful commitment to hemogenic endothelial precursors of the clonogenic CD34 hematopoietic cells. In contrast, the $2 \mathrm{D} /$ monolayer system displays a considerably lower level of endogenous Wnt/catenin (Figure 4) and therefore, a lower number of hematopoietic CD34 cells which lack clonogenic capacity. According to the results of 
this comparison, it can be hypothesized that the $2 \mathrm{D} /$ monolayer system deficiency results from its incapacity to respond adequately to BMP4 for complete mesoderm formation. To compensate for this weak potential, exogenous stimulation of Wnt signaling is practiced, but this directs differentiation to the definitive hematopoietic fate. Significantly, these deficiencies of the 2D format reflect the impossibility of such a format to model the unique, in vivo, 3D-like embryonic development. Meanwhile, the 2D format is capable to serve as a biotechnological platform for the production of hematopoietic cells useful for therapeutic purposes, in which cell-autonomous factors can operate only, leaving in an inactive state the integrative regulators of the developmental mechanisms.

Finally, this study shows the clear advantages of 3D culture as a basis for the advances in the understanding of the mechanistic aspects of in vivo hematopoietic events. Prospectively, this capacity of the 3D/EB format to model adequately the 3D niche condition for primitive hematopoiesis can also help to explain anomalies caused by mutations in the KDR and GATA2 genes, thus proposing an adequate in vitro system for drug testing $[52,53]$.

Supplementary Materials: The following are available online at https:/ /www.mdpi.com/article/10 .3390/cells10112858/s1, Figure S1: Pluripotency assessment of iPSCs in Monolayer and EB. Figure S2: Unstained controls and gating strategy for the comparison of EB/3D and 2D/Monolayer conditions. Figure S3: Unstained controls for the comparison of EB/3Dand2D/Monolayer conditions. Figure S4: Comparison of EB/3D and 2D/Monolayer conditions. Figure S5: Comparison of EB/3D and 2D/Monolayer conditions. Figure S6: Comparison of EB/3D and 2D/Monolayer conditions. Figure S7: Comparison of EB/3D and 2D/Monolayer conditions. Figure S8: Comparison of EB/3D and 2D/Monolayer conditions. Figure S9: Comparison of EB/3D and 2D/Monolayer conditions. Figure S10: Day 6 cells after 30 days of differentiation on AFT024 cells. Figure S11: 3D condition supports NK-like Phenotype when stimulated with CHIR99021. Table S1: Primers used for evaluation of mesodermal markers by qPCR, showing gene name, accesión number and secuences used, Table S2: List of antibodies used for flow cytometry and immunoflurorescence.

Author Contributions: G.A.M.-R. designed the experimental plan, performed the experiments, analyzed the data and wrote the manuscript; D.R.-R. performed flow cytometry acquisition and provided technical assistance in the analysis of the flow cytometry data; R.P. discussed the project idea and gave feedback of the results of hematopoietic aspects and K.G. proposed the idea of the project, participated in the discussion and in manuscript writing and edition. All authors have read and agreed to the published version of the manuscript.

Funding: The initial state of this work was supported by the Mexican National Council of Science and Technology (CONACYT, project number 201382, 2013-2015 for K.G.). DGAPA-PAPIIT AG200119 supported the late development of this project. G.A.M-R. received a scholarship from the National Council of Science and Technology (number 305949) and additional support from DGAPA-PAPIIT project ID AG200119.

Institutional Review Board Statement: Not applicable.

Informed Consent Statement: Not applicable.

Data Availability Statement: Not applicable.

Acknowledgments: The authors thank the participation of Ricardo Cevallos in the handling of the iPSCs line. We also thank Patricia Flores and Julieta Corral for technical assistance in CFU experiments. Vadim Perez Koldenkova, for the acquisition of the images of the spheres of the hPSCs. We also thank Arturo Hernandez for reviewing the manuscript. German Atzin Mora is a student from the Programa de Doctorado en Ciencias Biomedicas, Universidad Nacional Autonoma de Mexico (UNAM) and was supported by fellowship 305949 from CONACyT (Mexico).

Conflicts of Interest: The authors have no conflict to declare. 


\section{References}

1. Murry, C.E.; Keller, G. Differentiation of embryonic stem cells to clinically relevant populations: Lessons from embryonic development. Cell 2008, 132, 661-680. [CrossRef]

2. Ditadi, A.; Sturgeon, C.M. Directed differentiation of definitive hemogenic endothelium and hematopoietic progenitors from human pluripotent stem cells. Methods 2016, 101, 65-72. [CrossRef]

3. Sadlon, T.J.; Lewis, I.D.; D'Andrea, R.J. BMP4: Its role in development of the hematopoietic system and potential as a hematopoietic growth factor. Stem Cells 2004, 22, 457-474. [CrossRef]

4. Keller, G.; Kennedy, M.; Papayannopoulou, T.; Wiles, M.V. Hematopoietic commitment during embryonic stem cell differentiation in culture. Mol. Cell Biol. 1993, 13, 473-486. [CrossRef]

5. Kennedy, M.; D'Souza, S.L.; Lynch-Kattman, M.; Schwantz, S.; Keller, G. Development of the hemangioblast defines the onset of hematopoiesis in human ES cell differentiation cultures. Blood 2007, 109, 2679-2687. [CrossRef]

6. Crisan, M.; Kartalaei, P.S.; Vink, C.S.; Yamada-Inagawa, T.; Bollerot, K.; van IJcken, W.; van der Linden, R.; de Sousa Lopes, S.M.; Monteiro, R.; Mummery, C.; et al. BMP signalling differentially regulates distinct haematopoietic stem cell types. Nat. Commun. 2015, 6, 8040. [CrossRef]

7. D'Souza, S.S.; Maufort, J.; Kumar, A.; Zhang, J.; Smuga-Otto, K.; Thomson, J.A.; Slukvin, I.I. GSK3 $\beta$ Inhibition Promotes Efficient Myeloid and Lymphoid Hematopoiesis from Non-human Primate-Induced Pluripotent Stem Cells. Stem Cell Rep. 2016, 6, 243-256. [CrossRef] [PubMed]

8. de Bruijn, M. The hemangioblast revisited. Blood 2014, 124, 2472-2473. [CrossRef] [PubMed]

9. Lacaud, G.; Kouskoff, V. Hemangioblast, hemogenic endothelium, and primitive versus definitive hematopoiesis. Exp. Hematol. 2017, 49, 19-24. [CrossRef] [PubMed]

10. Huang, S.; Wang, J.; Liu, S.; Li, Y.; Hu, J.; Kou, Z.; Zhang, Y.; Sun, X.; Gao, S. Differentiation of reprogrammed somatic cells into functional hematopoietic cells. Differentiation 2009, 78, 151-158. [CrossRef] [PubMed]

11. Choi, K.; Kennedy, M.; Kazarov, A.; Papadimitriou, J.C.; Keller, G. A common precursor for hematopoietic and endothelial cells. Development 1998, 125, 725-732. [CrossRef]

12. Huber, T.L.; Kouskoff, V.; Fehling, H.J.; Palis, J.; Keller, G. Haemangioblast commitment is initiated in the primitive streak of the mouse embryo. Nature 2004, 432, 625-630. [CrossRef]

13. Knorr, D.A.; Ni, Z.; Hermanson, D.; Hexum, M.K.; Bendzick, L.; Cooper, L.J.; Lee, D.A.; Kaufman, D.S. Clinical-scale derivation of natural killer cells from human pluripotent stem cells for cancer therapy. Stem Cells Transl. Med. 2013, 2, 274-283. [CrossRef]

14. Niwa, A.; Heike, T.; Umeda, K.; Oshima, K.; Kato, I.; Sakai, H.; Suemori, H.; Nakahata, T.; Saito, M.K. A novel serum-free monolayer culture for orderly hematopoietic differentiation of human pluripotent cells via mesodermal progenitors. PLoS ONE 2011, 6, e22261. [CrossRef]

15. Niwa, A.; Umeda, K.; Chang, H.; Saito, M.; Okita, K.; Takahashi, K.; Nakagawa, M.; Yamanaka, S.; Nakahata, T.; Heike, T. Orderly hematopoietic development of induced pluripotent stem cells via Flk-1(+) hemoangiogenic progenitors. J. Cell. Physiol. 2009, 221, 367-377. [CrossRef] [PubMed]

16. Mills, J.A.; Paluru, P.; Weiss, M.J.; Gadue, P.; French, D.L. Hematopoietic differentiation of pluripotent stem cells in culture. Methods Mol. Biol. 2014, 1185, 181-194. [PubMed]

17. Galat, Y.; Dambaeva, S.; Elcheva, I.; Khanolkar, A.; Beaman, K.; Iannaccone, P.M.; Galat, V. Cytokine-free directed differentiation of human pluripotent stem cells efficiently produces hemogenic endothelium with lymphoid potential. Stem Cell Res. Ther. 2017, 8, 67. [CrossRef] [PubMed]

18. Ruiz, J.P.; Chen, G.; Haro Mora, J.J.; Keyvanfar, K.; Liu, C.; Zou, J.; Beers, J.; Bloomer, H.; Qanash, H.; Uchida, N.; et al. Robust generation of erythroid and multilineage hematopoietic progenitors from human iPSCs using a scalable monolayer culture system. Stem Cell Res. 2019, 41, 101600. [CrossRef] [PubMed]

19. Demirci, S.; Haro-Mora, J.J.; Leonard, A.; Drysdale, C.; Malide, D.; Keyvanfar, K.; Essawi, K.; Vizcardo, R.; Tamaoki, N.; Restifo, N.P.; et al. Definitive hematopoietic stem/progenitor cells from human embryonic stem cells through serum/feeder-free organoid-induced differentiation. Stem Cell Res. Ther. 2020, 11, 493. [CrossRef]

20. Nii, T.; Makino, K.; Tabata, Y. Three-Dimensional Culture System of Cancer Cells Combined with Biomaterials for Drug Screening. Cancers 2020, 12, 2754. [CrossRef]

21. Kaushik, G.; Ponnusamy, M.P.; Batra, S.K. Concise Review: Current Status of Three-Dimensional Organoids as Preclinical Models. Stem Cells 2018, 36, 1329-1340. [CrossRef]

22. Hernández-Sapiéns, M.A.; Reza-Zaldívar, E.E.; Cevallos, R.R.; Márquez-Aguirre, A.L.; Gazarian, K.; Canales-Aguirre, A.A. A Three-Dimensional Alzheimer's Disease Cell Culture Model Using iPSC-Derived Neurons Carrying A246E Mutation in PSEN1. Front. Cell. Neurosci. 2020, 14, 151. [CrossRef] [PubMed]

23. Olmer, R.; Haase, A.; Merkert, S.; Cui, W.; Palecek, J.; Ran, C.; Kirschning, A.; Scheper, T.; Glage, S.; Miller, K.; et al. Long term expansion of undifferentiated human iPS and ES cells in suspension culture using a defined medium. Stem Cell Res. 2010, 5, 51-64. [CrossRef] [PubMed]

24. Kwok, C.K.; Ueda, Y.; Kadari, A.; Günther, K.; Ergün, S.; Heron, A.; Schnitzler, A.C.; Rook, M.; Edenhofer, F. Scalable stirred suspension culture for the generation of billions of human induced pluripotent stem cells using single-use bioreactors. J. Tissue Eng. Regen. Med. 2018, 12, e1076-e1087. [CrossRef] [PubMed] 
25. Tian, X.F.; Heng, B.C.; Ge, Z.; Lu, K.; Rufaihah, A.J.; Fan, V.T.; Yeo, J.F.; Cao, T. Comparison of osteogenesis of human embryonic stem cells within 2D and 3D culture systems. Scand. J. Clin. Lab. Investig. 2008, 68, 58-67. [CrossRef]

26. Fong, A.H.; Romero-López, M.; Heylman, C.M.; Keating, M.; Tran, D.; Sobrino, A.; Tran, A.Q.; Pham, H.H.; Fimbres, C.; Gershon, P.D.; et al. Three-Dimensional Adult Cardiac Extracellular Matrix Promotes Maturation of Human Induced Pluripotent Stem Cell-Derived Cardiomyocytes. Tissue Eng. Part A 2016, 22, 1016-1025. [CrossRef] [PubMed]

27. Sacchetto, C.; Vitiello, L.; de Windt, L.J.; Rampazzo, A.; Calore, M. Modeling Cardiovascular Diseases with hiPSC-Derived Cardiomyocytes in 2D and 3D Cultures. Int. J. Mol. Sci. 2020, 21, 3404. [CrossRef]

28. Jeziorowska, D.; Fontaine, V.; Jouve, C.; Villard, E.; Dussaud, S.; Akbar, D.; Letang, V.; Cervello, P.; Itier, J.M.; Pruniaux, M.P.; et al. Differential Sarcomere and Electrophysiological Maturation of Human iPSC-Derived Cardiac Myocytes in Monolayer vs. Aggregation-Based Differentiation Protocols. Int. J. Mol. Sci. 2017, 18, 1173. [CrossRef]

29. Haraguchi, Y.; Matsuura, K.; Shimizu, T.; Yamato, M.; Okano, T. Simple suspension culture system of human iPS cells maintaining their pluripotency for cardiac cell sheet engineering. J. Tissue Eng. Regen. Med. 2015, 9, 1363-1375. [CrossRef]

30. Hamad, S.; Derichsweiler, D.; Papadopoulos, S.; Nguemo, F.; Šarić, T.; Sachinidis, A.; Brockmeier, K.; Hescheler, J.; Boukens, B.J.; Pfannkuche, K. Generation of human induced pluripotent stem cell-derived cardiomyocytes in 2D monolayer and scalable 3D suspension bioreactor cultures with reduced batch-to-batch variations. Theranostics 2019, 9, 7222-7238. [CrossRef]

31. Choi, K.-D.; Vodyanik, M.A.; Slukvin, I.I. Generation of mature human myelomonocytic cells through expansion and differentiation of pluripotent stem cell-derived lin-CD34+CD43+CD45+ progenitors. J. Clin. Investig. 2009, 119, 2818-2829. [CrossRef]

32. Yanagimachi, M.D.; Niwa, A.; Tanaka, T.; Honda-Ozaki, F.; Nishimoto, S.; Murata, Y.; Yasumi, T.; Ito, J.; Tomida, S.; Oshima, K.; et al. Robust and highly-efficient differentiation of functional monocytic cells from human pluripotent stem cells under serumand feeder cell-free conditions. PLoS ONE 2013, 8, e59243. [CrossRef]

33. Ohta, R.; Niwa, A.; Taniguchi, Y.; Suzuki, N.M.; Toga, J.; Yagi, E.; Saiki, N.; Nishinaka-Arai, Y.; Okada, C.; Watanabe, A.; et al. Laminin-guided highly efficient endothelial commitment from human pluripotent stem cells. Sci. Rep. 2016, 6, 35680. [CrossRef]

34. Cevallos, R.R.; Rodríguez-Martínez, G.; Gazarian, K. Wnt/ $\beta$-Catenin/TCF Pathway Is a Phase-Dependent Promoter of Colony Formation and Mesendodermal Differentiation During Human Somatic Cell Reprogramming. Stem Cells 2018, 36, 683-695. [CrossRef]

35. Ramirez-Ramirez, D.; Vadillo, E. Early Differentiation of Human CD11c(+)NK Cells with gammadelta T Cell Activation Properties Is Promoted by Dialyzable Leukocyte Extracts. J. Immunol. Res. 2016, 2016, 4097642. [CrossRef]

36. Pfaffl, M.W. A new mathematical model for relative quantification in real-time RT-PCR. Nucleic Acids Res. 2001, 29, e45. [CrossRef]

37. Gadue, P.; Huber, T.L.; Paddison, P.J.; Keller, G.M. Wnt and TGF-beta signaling are required for the induction of an in vitro model of primitive streak formation using embryonic stem cells. Proc. Natl. Acad. Sci. USA 2006, 103, 16806-16811. [CrossRef]

38. Loh, K.M.; Chen, A.; Koh, P.W.; Deng, T.Z.; Sinha, R.; Tsai, J.M.; Barkal, A.A.; Shen, K.Y.; Jain, R.; Morganti, R.M.; et al. Mapping the Pairwise Choices Leading from Pluripotency to Human Bone, Heart, and Other Mesoderm Cell Types. Cell 2016, 166, 451-467. [CrossRef] [PubMed]

39. Nostro, M.C.; Cheng, X.; Keller, G.M.; Gadue, P. Wnt, activin, and BMP signaling regulate distinct stages in the developmental pathway from embryonic stem cells to blood. Cell Stem Cell 2008, 2, 60-71. [CrossRef] [PubMed]

40. Richter, J.; Traver, D.; Willert, K. The role of Wnt signaling in hematopoietic stem cell development. Crit. Rev. Biochem. Mol. Biol. 2017, 52, 414-424. [CrossRef] [PubMed]

41. Sturgeon, C.M.; Ditadi, A.; Awong, G.; Kennedy, M.; Keller, G. Wnt signaling controls the specification of definitive and primitive hematopoiesis from human pluripotent stem cells. Nat. Biotechnol. 2014, 32, 554-561. [CrossRef]

42. Sumi, T.; Tsuneyoshi, N.; Nakatsuji, N.; Suemori, H. Defining early lineage specification of human embryonic stem cells by the orchestrated balance of canonical Wnt/beta-catenin, Activin/Nodal and BMP signaling. Development 2008, 135, 2969-2979. [CrossRef]

43. Clarke, R.L.; Yzaguirre, A.D.; Yashiro-Ohtani, Y.; Bondue, A.; Blanpain, C.; Pear, W.S.; Speck, N.A.; Keller, G. The expression of Sox17 identifies and regulates haemogenic endothelium. Nat. Cell Biol. 2013, 15, 502-510. [CrossRef] [PubMed]

44. Kyba, M. Hemogenic endothelium in a dish. Blood 2013, 121, 417-418. [CrossRef] [PubMed]

45. Nakajima-Takagi, Y.; Osawa, M.; Oshima, M.; Takagi, H.; Miyagi, S.; Endoh, M.; Endo, T.A.; Takayama, N.; Eto, K.; Toyoda, T.; et al. Role of SOX17 in hematopoietic development from human embryonic stem cells. Blood 2013, 121, 447-458. [CrossRef] [PubMed]

46. Takahashi, S.; Nobuhisa, I.; Saito, K.; Gerel, M.; Itabashi, A.; Harada, K.; Osawa, M.; Endo, T.A.; Iwama, A.; Taga, T. Sox17mediated expression of adherent molecules is required for the maintenance of undifferentiated hematopoietic cluster formation in midgestation mouse embryos. Differentiation 2020, 115, 53-61. [CrossRef]

47. Sakurai, H.; Era, T.; Jakt, L.M.; Okada, M.; Nakai, S.; Nishikawa, S.; Nishikawa, S. In vitro modeling of paraxial and lateral mesoderm differentiation reveals early reversibility. Stem Cells 2006, 24, 575-586. [CrossRef]

48. Wang, P.; Rodriguez, R.T.; Wang, J.; Ghodasara, A.; Kim, S.K. Targeting SOX17 in human embryonic stem cells creates unique strategies for isolating and analyzing developing endoderm. Cell Stem Cell 2011, 8, 335-346. [CrossRef]

49. Galat, Y.; Elcheva, I.; Dambaeva, S.; Katukurundage, D.; Beaman, K.; Iannaccone, P.M.; Galat, V. Application of small molecule CHIR99021 leads to the loss of hemangioblast progenitor and increased hematopoiesis of human pluripotent stem cells. Exp. Hematol. 2018, 65, 38-48.e1. [CrossRef] 
50. Kang, H.; Mesquitta, W.-T.; Jung, H.S.; Moskvin, O.V.; Thomson, J.A.; Slukvin, I.I. GATA2 Is Dispensable for Specification of Hemogenic Endothelium but Promotes Endothelial-to-Hematopoietic Transition. Stem Cell Rep. 2018, 11, 197-211. [CrossRef]

51. Slukvin, I.I. Deciphering the hierarchy of angiohematopoietic progenitors from human pluripotent stem cells. Cell Cycle 2013, 12, 720-727. [CrossRef] [PubMed]

52. Jung, M.; Cordes, S.; Zou, J.; Yu, S.J.; Guitart, X.; Hong, S.G.; Dang, V.; Kang, E.; Donaires, F.S.; Hassan, S.A.; et al. GATA2 deficiency and human hematopoietic development modeled using induced pluripotent stem cells. Blood Adv. 2018, 2, 3553-3565. [CrossRef] [PubMed]

53. Oszajca, K.; Szemraj, J.; Wyrzykowski, D.; Chrzanowska, B.; Salamon, A.; Przewratil, P. Single-nucleotide polymorphisms of VEGF-A and VEGFR-2 genes and risk of infantile hemangioma. Int. J. Dermatol. 2018, 57, 1201-1207. [CrossRef] [PubMed] 